\title{
Blocking and partial reinforcement: Effects of $N-R$ transitions early vs. late in training on resistance to extinction
}

\author{
STEVEN J. HAGGBLOOM \\ Arkansas State University, State University, Arkansas 72467
}

\begin{abstract}
In Experiment 1, two groups of rats $(\mathrm{n}=10)$ received the same schedules of partial reinforcement in acquisition. The schedules were arranged over days so that, for Group Early, most (12) of the nonrewarded to rewarded (N-R) trial transitions occurred in the first half of acquisition and only a few (4) occurred in the second half. Those conditions were reversed for Group Late. In Experiment 2, ( $n=8$ rats/group), two groups experienced the early conditions and two groups experienced the late conditions employed in Experiment 1. Groups Early: L-S and Late: L-S received large reward during the first half of acquisition and small reward during the second half. The reward conditions were reversed for Groups Early: S-L and Late: S-L. In Experiment 1, Group Early was more resistant to extinction than Group Late. In Experiment 2, resistance to extinction was greater in Group Early: L-S than in Group Late: L-S, but Group Late: S-L was more resistant to extinction than Group Early: S-L. These results were predicted from implications of blocking experiments for partial reinforcement phenomena.
\end{abstract}

Blocking experiments (e.g., Kamin, 1969) emphasize the retarding effects of prior conditioning to one stimulus (A) on learning about another stimulus (X) that occurs only as part of an AX stimulus compound. Most blocking experiments have employed conventional, exteroceptive stimuli (e.g., lights, tones). Partial reinforcement experiments emphasize the acquisition of behavior control by interoceptive stimuli produced by nonreward (Amsel, 1958; Capaldi, 1966).

Recent experiments indicate that interoceptive and exteroceptive stimuli enter into stimulus compounds (e.g., Haggbloom \& Tillman, 1980) and that prior conditioning to interoceptive cues blocks conditioning to exteroceptive cues (Capaldi, Verry, \& Nawrocki, 1982; Haggbloom 1981) and vice versa (Haddad, Walkenbach, Preston, \& Strong, 1981). Implications of these blocking experiments for partial reinforcement phenomena have not been investigated.

One implication of blocking for partial reinforcement situations, investigated in the two experiments reported here, is that resistance to extinction should be reduced in a group given nonrewarded $(\mathrm{N})$ to rewarded (R) trial transitions (N-R transitions) late in training relative to a group given the same number of N-R transitions early in training. N-R transitions condition responding to nonreward-produced interoceptive stimuli $\left(\mathrm{SN}_{\mathrm{S}}\right)$.

These experiments were supported by a grant to the author from Arkansas State University. The author wishes to thank John K. Beadles for the use of laboratory space in the Department of Biology. Requests for reprints should be addressed to Steven J. Haggbloom, Department of Psychology, Arkansas State University, P.O. Box 2127, State University, Arkansas 72467.
Since $\mathrm{S}^{\mathrm{N}}$ extensively regulates extinction behavior, $\mathrm{N}-\mathrm{R}$ transitions constitute one of the major variables influencing resistance to extinction (Capaldi, 1966).

During partial reinforcement training, $S^{N}$ necessarily occurs in compound with exteroceptive (e.g., runway and background) stimuli. If occasions for conditioning responding to $\mathrm{S}^{\mathrm{N}}$ (i.e., N-R transitions) occur late in training, prior conditioning to exteroceptive cues should block conditioning to $\mathrm{SN}$. Blocking in this instance would result in a reduction in resistance to extinction.

Experiment 1 here investigated the effects of N-R transitions early vs. late in training on resistance to extinction. Groups Early and Late received the same partial reinforcement schedules over the course of acquisition and the same number of $\mathrm{R}$ and $\mathrm{N}$ trials on each day of training. For Group Early, the schedules were arranged over days so that most (12) of the N-R transitions occurred early in training and only a few (4) occurred late in training. The opposite arrangement was employed for Group Late.

Experiment 2 investigated the joint effects on resistance to extinction of early vs. late N-R transitions and a change in reward magnitude between the early and late stages of training. Changes in reward magnitude attenuate blocking in both Pavlovian (Kamin, 1969; Mackintosh \& Turner, 1971) and instrumental (Neely \& Wagner, 1974; Haggbloom, Note 1) conditioning. The early and late conditions in Experiment 2 were identical to those in Experiment 1 . One group given early $\mathrm{N}-\mathrm{R}$ transitions and one group given late $N-R$ transitions received large reward (L) early in training followed by small reward (S) late in training (Groups Early: L-S and Late: L-S). The remaining two groups received $S$ early in training and L late in training (Groups Early: S-L and Late: S-L). 


\section{METHOD}

Subjects

The subjects in both experiments were male rats, 90 days old, bred in the laboratory from Holtzman stock. In Experiment 1,10 rats were randomly assigned to both groups. In Experiment 2, eight rats were randomly assigned to each of four groups.

\section{Apparatus}

The apparatus, used in both experiments, consisted of a straight black runway, $130 \mathrm{~cm}$ long $\times 9 \mathrm{~cm}$ high and wide. The first $25 \mathrm{~cm}$ made up the startbox, and the last $25 \mathrm{~cm}$ constituted the goalbox. The startbox was separated from the runway by a manually operated guillotine door. The goalbox, separated from the runway in the same manner, contained an unpainted wooden goal cup. Start times were recorded from the opening of the startbox door, which triggered a $.01-\mathrm{sec}$ clock, to a point $30 \mathrm{~cm}$ into the runway. Run and goal times were measured over the next 40 and $30 \mathrm{~cm}$, respectively. The offset of the first clock and the operation of the run and goal clocks were controlled by photoelectric circuitry.

\section{Procedure}

All food was removed from each rat's cage on Day 1 . Thereafter, each rat received $12 \mathrm{~g}$ of lab chow per day minus the amount of food consumed in the runway. Water was always available. On Days 12-14 of both experiments, the rats were handled in squads of four for $4 \mathrm{~min} / \mathrm{squad}$.

Five trials were administered on each of 12 acquisition and 3 extinction days, beginning on Day 15, in both experiments. The daily reward schedules used in acquisition comprised two 6-day series, Series A-RNRNR, RNRNR, NRNNR, RNRNR, NRRNR, NRRNR, and Series B-RRRNN, RRRNN, NRRNN, RNRRN, NRRRN, NRRRN. The two series contain 12 and $4 \mathrm{~N}-\mathrm{R}$ transitions, respectively. Group Early in Experiment 1 and Groups Early: L-S and Early: S-L in Experiment 2 received the two series in the order AB. Group Late in Experiment 1 and Groups Late: L-S and Late: S-L received the two series in the order BA.

Reinforcement in Experiment 1 consisted of 1045 -mg Noyes pellets. In Experiment 2, Groups Early: L-S and Late: L-S received $1645-\mathrm{mg}$ pellets on $\mathrm{R}$ trials during training on the series (A or B) received first and 2 pellets during training on the second series. Groups Early: S-L and Late: S-L received 2 pellets during training on the first series and 16 pellets during the second series. In both experiments, rats were confined to the unbaited goalbox for $20 \mathrm{sec}$ on $\mathrm{N}$ trials.

The subjects were run in squads of four containing two subjects from both groups (Experiment 1) or one subject from each group (Experiment 2). In both experiments, each rat within a squad received Trial 1 before any rat received Trial 2, and so on. The order of running subjects within a squad was randomized daily, and the order of successive squads was held constant across days. In both experiments, the intertrial interval in acquisition and extinction was 3-5 min.

All trials were initiated by opening the startbox door approximately $3 \mathrm{sec}$ after placing the rat in the startbox and regardless of the rat's orientation. A maximum time of $30 \mathrm{sec}$ was allowed in each section of the alley. If $30 \mathrm{sec}$ was exceeded in any alley section, the additional time was added to the time score of the next section forward. If the animal did not enter the goalbox within $90 \mathrm{sec}$, it was placed in the goalbox.

\section{RESULTS}

In both experiments, the times for each subject on each trial were converted to speeds in centimeters per second. One rat in Group Early was dropped from
Experiment 1 for refusing to run in acquisition. In Experiment 1, an analysis of variance (ANOVA) with groups (two) as a between-subjects variable and trials (five) as a within-subjects variable was applied to speeds over the total length of the alley on the last day of acquisition. A similar ANOVA, with the addition of days (three) as a within-subjects variable, was applied to total speeds in extinction. Identical ANOVAs were employed in Experiment 2, except (1) the between-subjects variable was partitioned into early vs. late N-R transitions (two) and reward magnitude early and late in training (two) and (2) results for the goal section are reported for Experiment 2 (the relationship among groups in resistance to extinction was the same in each alley section, but the expected interaction between early vs. late $\mathrm{N}-\mathrm{R}$ transitions and reward magnitude was reliable only in the goal section).

\section{Experiment 1}

Figure 1 shows the speed of running for both groups over the total alley on the last day of acquisition and on each of the five daily extinction trials collapsed across the 3 days of extinction. As can be seen, there was no difference between Groups Early and Late at the end of acquisition ( $F s<1$ for both the groups effect and the Groups by Trials interaction). In extinction, running speeds declined more rapidly over daily trials in Group Late than in Group Early. The extinction ANOVA yielded significant effects for both groups $[F(1,17)=$ 7.07, $\mathrm{p}<.05$ ] and the Groups by Trials interaction $[\mathrm{F}(4,68)=4.92, \mathrm{p}<.01]$.

\section{Experiment 2}

In acquisition (data not shown), groups given $\mathrm{L}$ on Days 1-6 showed a faster rate of acquisition than groups given $S$. The shift from $L$ to $S$ did not reduce running speeds in Groups Early: L-S and Late: L-S, but those groups did run slower over Days 7-12 than Groups Early: S-L and Late: S-L. Figure 2 shows running speeds

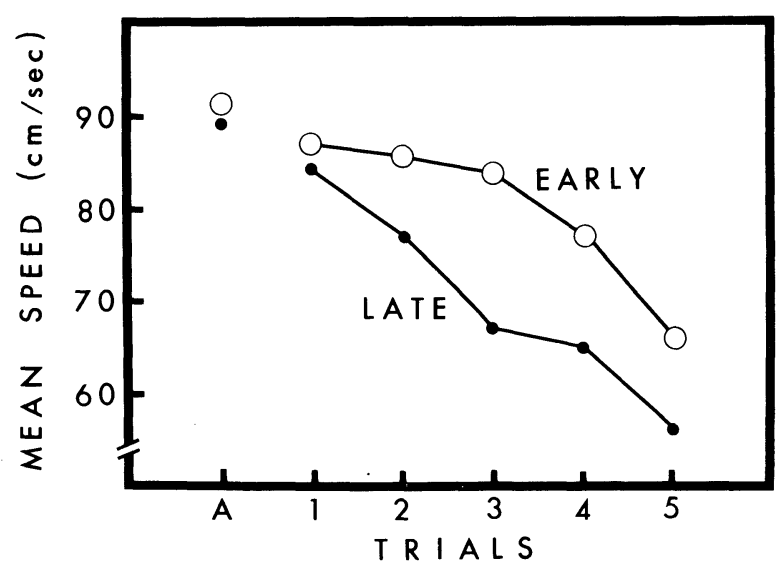

Figure 1. Mean total speed of both groups in Experiment 1 on the last day of acquisition (A) and on each of the five daily extinction trials collapsed across the 3 days of extinction. 




Figure 2. Mean speed in the goal section for each of the four groups in Experiment 2 on the last day of acquisition (A) and on each of the five daily extinction trials collapsed across the 3 days of extinction.

in the goal section for each of the four groups on the last day of acquisition and on each of the five daily extinction trials collapsed across the 3 days of extinction. At the end of acquisition, groups given $L$ late in training ran faster than groups given $S$ late in training $[F(1,28)=$ $17.72, \mathrm{p}<.01]$, but there were no differences in speed of running as a function of early vs. late N-R transitions $(F<1)$, nor did that variable interact with reward magnitude $(\mathrm{F}<1)$.

As can be seen in Figure 2, resistance to extinction was increased by early $\mathrm{N}-\mathrm{R}$ transitions over late $\mathrm{N}-\mathrm{R}$ transitions in groups given $\mathrm{L}$ early in training (Group Early: L-S vs. Group Late: L-S), but the reverse was true in groups given $L$ late in training (Group Late: S-L vs. Group Early: S-L). This resulted in a reliable Early vs. Late N-R Transitions by Reward Magnitude by Trials interaction $[F(4,112)=2.60, p<.05]$. Simple interactions of Early vs. Late N-R Transitions by Trials supported the observation that extinction occurred at a faster rate in Group Late: L-S than in Group Early: L-S $[F(4,112)=2.69, \mathrm{p}<.05]$ and at a faster rate in Group Early: S-L than in Group Late: S-L $[\mathrm{F}(4,112)=2.84$, $\mathrm{p}<.05$ ]

\section{DISCUSSION}

As predicted, resistance to extinction in Experiment 1 was reduced in a partial reinforcement group given N-R transitions primarily late in training relative to a group given the same reward schedules but arranged so that $N-R$ transitions occurred primarily early in training. Experiment 2 showed that the effect of early vs. late N-R transitions obtained in Experiment 1 was not per se a function of different ordering of the reward schedules for those conditions; rather, it depended upon whether circumstances favored stronger conditioning to $\mathrm{SN}$ early or late in training. The analogy to a model of blocking like that proposed by Wagner and Rescorla (1972) suggested that, under the constant reward magnitude condition in Experiment 1, stronger conditioning to $\mathrm{S}^{\mathrm{N}}$ would occur early in training than late in training. This is true as well for groups given $\mathrm{L}$ early in training and $S$ late in training in Experiment 2. Thus, Group Early: L-S was more resistant to extinction than Group Late: L-S. However, in groups given $\mathrm{S}$ early in training and $\mathrm{L}$ late in training, conditions would be favorable for conditioning to $S^{N}$ late in training. Although exteroceptive cues would acquire control early in training, the asymptote of conditioning under $\mathrm{S}$ would be low. The substantially higher asymptote of conditioning under $\mathrm{L}$ late in training would allow conditioning to occur to $S^{N}$ in the same way that a shift in reward magnitude attenuates blocking (see, e.g., Kamin, 1969). Thus, Group Late: S-L was more resistant to extinction than Group Early: S-L.

It should be emphasized that no claim is being made here that the present results constitute a demonstration of blocking. Rather, blocking may provide a useful way of looking at some partial reinforcement phenomena. Other interpretations, though, are certainly possible. For example, reinforcement-level theory (Capaldi, Note 2) can explain the present results by appealing to changes in expectancy of reward, and resultant changes in the strength of conditioning, over the course of training. Essentially the same assumptions could be applied to the present case as Capaldi (1974) employed to explain the effects of partial (P) and consistent (C) reward schedules in tandem (i.e., $\mathrm{P}-\mathrm{C}$ vs. C-P) on resistance to extinction. Indeed, the P-C vs. C-P manipulation can be viewed as the limiting case of the present manipulation of N-R transitions early vs. late in training.

On the other hand, the P-C vs. C-P effect is amenable to a blocking interpretation. That is, one could argue that C-P training generally produces less resistance to extinction than $\mathrm{P}-\mathrm{C}$ training because, under C-P training, $S N$ is introduced late and after substantial conditioning has already occurred to exteroceptive cues. Nor are explanations of the P-C vs. C-P effect and early vs. late $\mathrm{N}-\mathrm{R}$ transition effect in terms of reinforcementlevel theory or blocking mutually exclusive. The stimulus compounding assumptions of the Rescorla-Wagner model are compatible with the reinforcement-level principle, as, indeed, Capaldi (Note 2) indicated.

\section{REFERENCE NOTES}

1. Haggbloom, S. J. Attenuation of blocking in instrumental conditioning by a shift in reinforcement magnitude. Paper presented to the meeting of the Southwestern Psychological Association, Dallas, Texas, April 1982.

2. Capaldi, E. J. Reinforcement level theory: An expectancyassociative approach to relative reinforcement and nonreinforcement effects. In the Arlington Symposium on Learning. Symposium presented at the University of Texas at Arlington, February 1975.

\section{REFERENCES}

Amsel, A. The role of frustrative nonreward in noncontinuous reward situations. Psychological Bulletin, 1958, 55, 102-119.

Capaldi, E. J. Partial reinforcement: An hypothesis of sequential effects. Psychological Review, 1966, 73, 459-477.

Capaldi, E. J. Partial reward either following or preceding consistent reward: A case reinforcement level. Journal of Experimental Psychology, 1974, 102, 954-962.

Capaldi, E. J., Verry, D. R., \& Nawrocki, T. M. Alley section effects on blocking. Bulletin of the Psychonomic Society, 1982, 20, 109-111.

Haddad, N. F., Walkenbach, J., Pregton, M., Strona, R. Stimulus control in a simple instrumental task: The role of internal and external stimuli. Learning and Motivation, 1981, 12, 509-520.

НАасвLOом, S. J. Blocking in successive differential conditioning: Prior acquisition of control by internal cues blocks the acquisition of control by brightness. Learning and Motivation, $1981,12,485-508$. 
Hagabloom, S. J., \& Tillman, D. J. Sequential effects on discrimination reversal. Learning and Motivation, 1980, 11, 318338.

Kamin, L. J. Predictability, surprise, attention and conditioning. In B. Campbell \& R. Church (Eds.), Punishment and aversive behavior. New York: Appleton-Century-Crofts, 1969.

Mackintosh, N. J., \& TURner, C. Blocking as a function of novelty of CS and predictability of UCS. Quarterly Journal of Experimental Psychology, 1971, 23, 359-366.

NeELY, J. J., \& WAGNER, A. R. Attenuation of blocking with shifts in reward: The involvement of schedule-generated contextual cues. Journal of Experimental Psychology, 1974, 102, 751-763.

Whaner, A. R., \& Rescorla, R. A. Inhibition in Pavlovian conditioning: Application of a theory. In R. A. Boakes \& M. S. Halliday (Eds.), Inhibition and learning. New York: Academic Press, 1972.

(Received for publication January 10, 1983.) 